\title{
Questes
}

vestes Revue pluridisciplinaire d'études médiévales

Violences médiévales

\section{Sur les traces de la bataille de Visby}

\section{Renaud Figueres}

\section{(2) OpenEdition \\ Journals}

\section{Édition électronique}

URL : http://journals.openedition.org/questes/397

DOI : 10.4000/questes.397

ISSN : 2109-9472

\section{Éditeur}

Les Amis de Questes

\section{Édition imprimée}

Date de publication : 15 mars 2008

Pagination : 22-37

ISSN : 2102-7188

\section{Référence électronique}

Renaud Figueres, "Sur les traces de la bataille de Visby », Questes [En ligne], 14 | 2008, mis en ligne le 01 janvier 2014, consulté le 30 avril 2019. URL : http://journals.openedition.org/questes/397 ; DOI :

10.4000/questes.397 


\title{
Sur les traces de la bataille de Visby
}

\author{
Renaud FIGUERES
}

Sur l'île de Gotland (à l'est de la Suède), le 27 juillet 1361, une armée sous équipée et mal entraînée allait défendre sa ville et sa terre contre l'invasion d'un corps expéditionnaire mené par le roi du Danemark. Fait rarissime, les traces du massacre qui allait suivre sont parvenues jusqu'à nous, le site de la bataille ayant fait l'objet de fouilles au début du siècle.

Si les sources contemporaines nous ont laissé peu de choses sur le déroulement de l'affrontement, on sait cependant qu'il se solda par la défaite et le massacre des Suédois. Mais le caractère unique de cette bataille ne réside pas là. Après tout, la clémence, dans les conflits médiévaux, ne concernait que les puissants et la piétaille payait souvent au prix fort les conséquences de la défaite. À ce titre, la bataille de Visby n'en est qu'une des nombreuses illustrations. Son caractère unique vient du fait que sont parvenus jusqu'à nous les charniers, les sépultures des combattants, enterrés hâtivement à l'orée de la ville (près de 1200 corps, répartis sur plusieurs fosses). Fait plus rare encore, les habitants de Visby n'ont pas pris la peine de dépouiller certains corps (illustration 1) et les ont enterrés avec ce qu'ils avaient sur eux (illustration 2). 


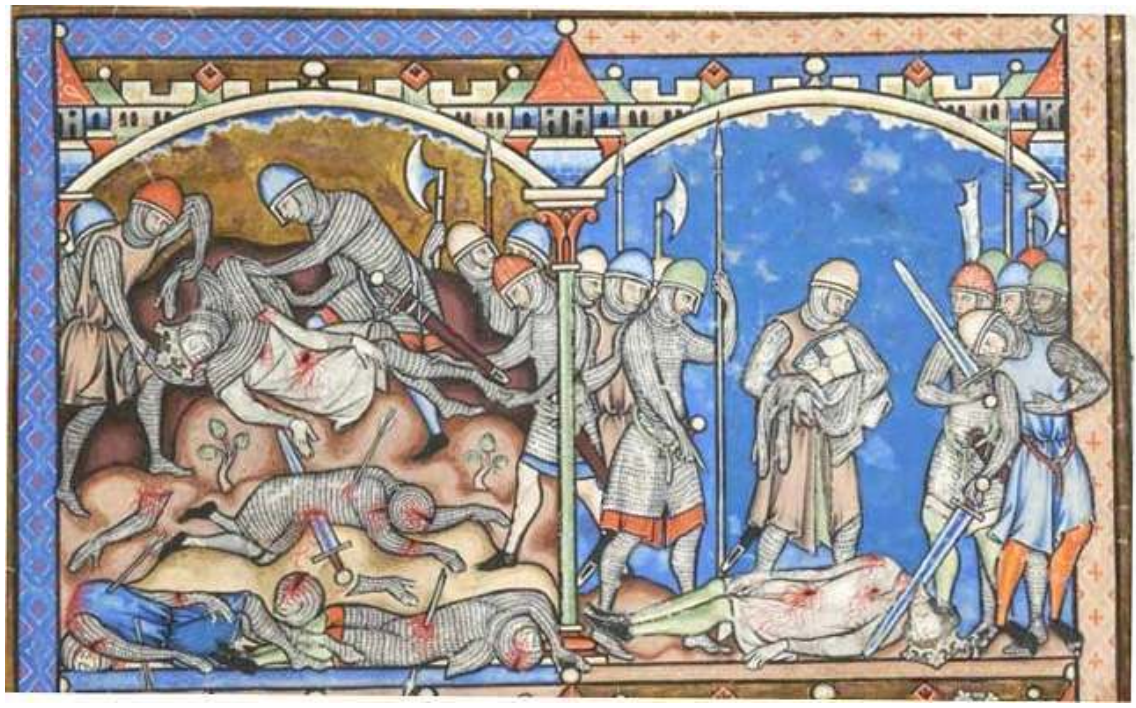

Illustration 1 : Bible de Maciejowski, New York, Pierpont Morgan Library, MS. M. 638, $\mathrm{f}^{\circ} 35 \mathrm{r}^{\circ}$

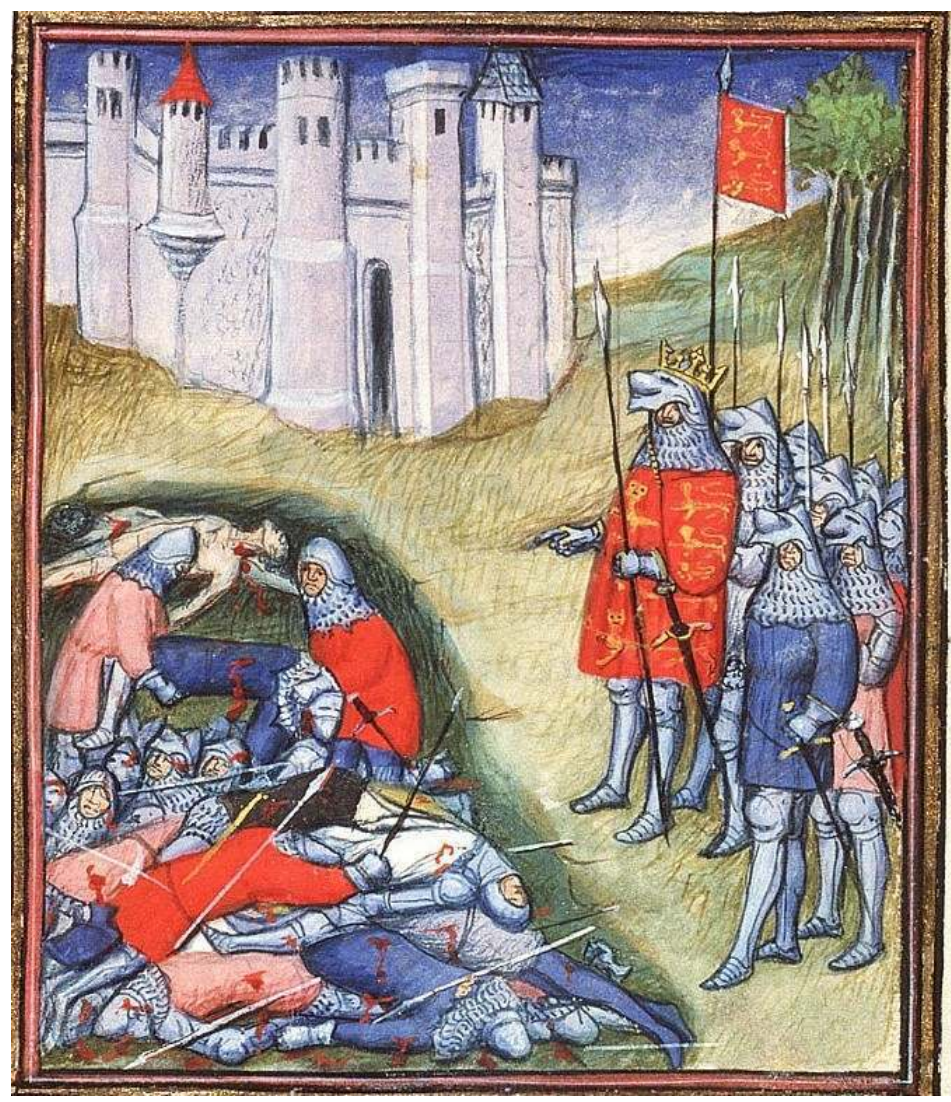

Illustration 2 : Edouard III comptant les morts après la bataille de Crécy. Chroniques de Froissart, La Haye, Koninklijke Bibliotheek, KB 72 A 25, $f^{\circ} 144 r^{\circ}$ 
Parmi les nombreux artefacts mis au jour lors des fouilles, les éléments d'armures sont sans doute les plus intéressants car les plus rares. Aucun casque, bouclier ou arme de guerre (si l'on excepte les fers de traits) ne fut retrouvé.

Ces découvertes permirent non seulement d'établir un parallèle entre des armures de la seconde moitié de $\mathrm{XIV}^{\mathrm{e}}$ (trouvées en contexte, c'est-à-dire en condition de fouille) et certaines enluminures contemporaines, mais également d'apporter, dans une certaine mesure, un nouvel éclairage sur ce que nous disent les textes.

Enfin, la découverte de ces charniers a offert pour la première fois la possibilité de voir de visu les effets (du moins, certains d'entre eux) des armes sur les combattants médiévaux.

\section{$* * * *$}

Nous aborderons dans un premier temps la question des armures, des armes, puis des rapprochements que l'on peut faire avec quelques manuscrits contemporains avant de terminer par quelques données et considérations sur l'efficacité des armes médiévales.

Sur au moins 1185 combattants inhumés, une vingtaine d'armures ont été retrouvées (et certaines reconstituées, illustration 3). 

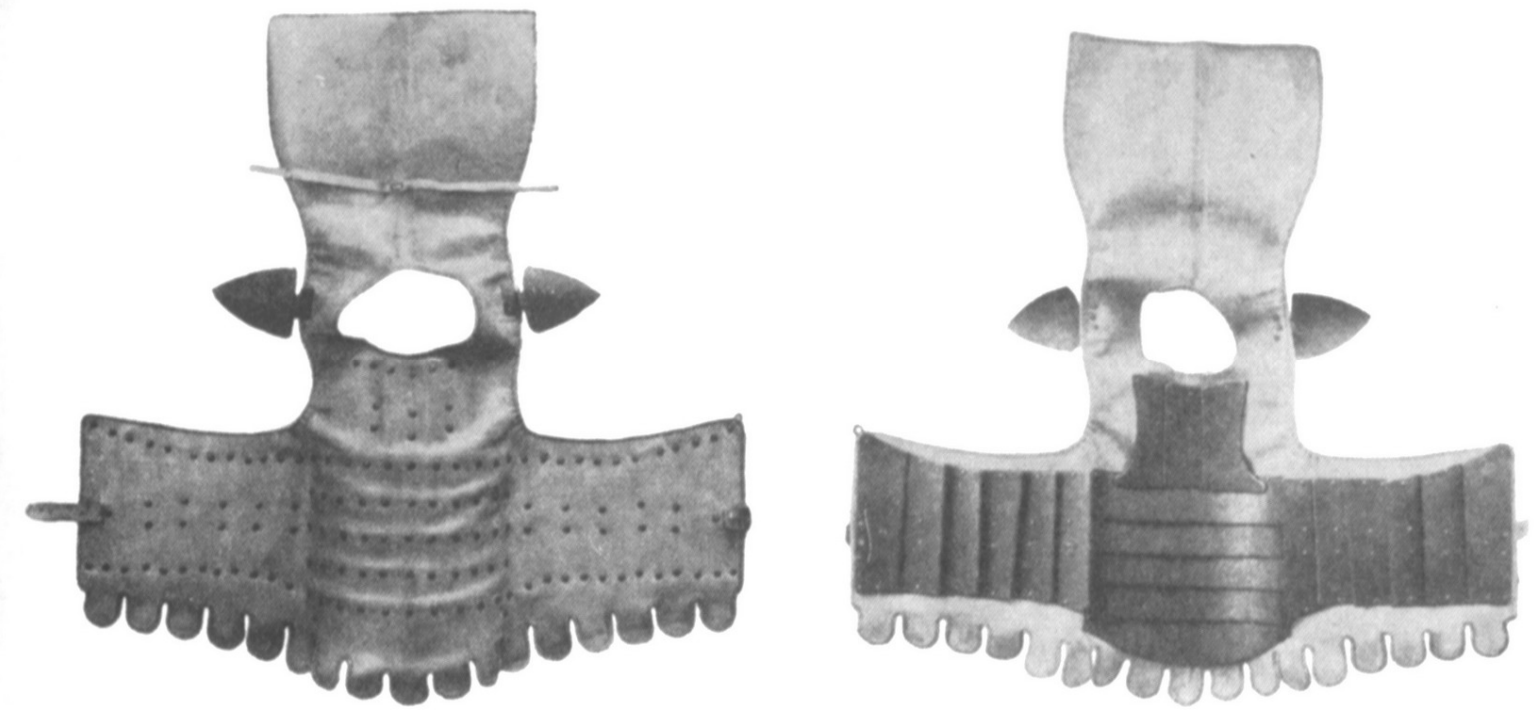

Illustration 3 : reconstitution d'une armure (Thordeman, 2001) ${ }^{1}$

Même si les armures retrouvées à Visby appartiennent toutes (à l'exception d'une seule) à la famille des «cottes de plaques », c'est-à-dire constituées de plaques de métal rivées à un support de cuir ou de tissu, elles diffèrent dans leur mode de construction. En fait, pas un modèle n'a été construit de manière identique. On retrouvera cette diversité de confection pour les cottes de mailles et les gantelets.

Des plates métalliques recouvrant les membres, peu de traces. Si quelques unes furent bien retrouvées, aucune de type grèves ou canons ne fut mise au jour.

Outre les armures, on retrouva de nombreux camails (185, dans quatre cas, les camails furent retrouvés associés à un squelette en armure) et une quinzaine d' haubergeons (chemise de maille, plus courte que le haubert, et couvrant le torse et les bras), souvent très altérés. Des gantelets furent également mis au jour. Assez bien conservées, les plaques qui les composent sont de structure étonnamment fine : ces gantelets devaient être d'une protection toute relative face à un coup direct.

\footnotetext{
${ }^{1}$ Bengt ThORDEMAn, Armour from the Battle of Wisby, 1361, Hardcover, Chivalry Bookshelf, 2001, p. 219.
} 
Mais toutes les protections n'étaient pas en métal. Ainsi, le gambison était un veston rembourré dont le rôle était d'atténuer le choc et d'absorber, du moins en partie, l'énergie cinétique d'un coup (la maille ou la plaque étant censée, elle, neutraliser le pouvoir de coupe et de pénétration). Cette protection était souvent recouverte et apparaît donc rarement dans les enluminures. Si on n'en a retrouvé aucun exemplaire, il devait néanmoins être présent car c'était un équipement de base pour le combattant.

En ce qui concerne les armes, on sait peu de choses. D'après les chroniques, on sait que les Danois disposaient d'arbalétriers et il est possible qu'il y ait eu des archers de part et d'autres. Quoiqu'il en soit, ces armes ont été massivement employées si l'on en croit les nombreuses traces de blessures occasionnées par ce type d'arme ainsi que les nombreux fers de trait retrouvés.

Concernant les armes de mêlées, il faut d'abord remarquer que, si cette époque est charnière pour les armures, elle l'est aussi pour les armes.

Ainsi, l'usage des plates se démocratisant, les épées voient leurs pointes s'effiler et se renforcer, permettant de pratiquer l'estoc contre des adversaires de moins en moins vulnérables aux coups de tailles. Mais qu'elles aient été archaïques ou plus modernes, les épées furent sans doute les armes les plus présentes sur le champ de bataille.

Les haches ont également dû être utilisées, notamment celles connues sous le nom de "haches danoises " (à long manche et large fer, elles se maniaient à deux mains). Les armes contondantes (masses d'armes, marteaux de guerre) étaient aussi probablement présentes, même si elles ont laissées moins de traces.

Quant aux armes d'hast, rares sont les batailles médiévales où elles n'ont pas eu leurs places. La lance par exemple, a toujours été une arme populaire car bon marché et très efficace, à tel point qu'un ouvrage norvégien du 
milieu du XIII ${ }^{\mathrm{e}}$ siècle, le Speculum Regale estimait qu'" à la guerre, une lance vaut plus que deux épées $»^{2}$.

\section{$* * * *$}

Les découvertes que l'on vient de voir permirent de faire des rapprochements avec les sources déjà existantes, à commencer par les enluminures. Mais le spectre des représentations d'armes et d'armures dans les manuscrits à peintures est très large, allant du meilleur au pire. Et, bien qu'ils représentent une source documentaire non négligeable, la principale difficulté (du point de vue archéologique) a toujours été de savoir quel était le véritable degré de fiabilité des équipements représentés par l'artiste.

De plus, l'illustration n'a pas, dans l'immense majorité des cas, de valeur «documentaire» et il est souvent difficile de faire la part entre le figuratif, le symbolique et l'imaginaire. Dans certains cas, il est évident que l'auteur n'a jamais été en contact avec les éléments qu'il dépeint. Mais les armures de Visby permirent de montrer que cela pouvait également être le contraire.

Deux manuscrits furent particulièrement précieux pour effectuer ces comparaisons. Le premier contient La Romance d'Alexandre, et est aujourd'hui conservé à Oxford (Oxford, Bodleian Library, ms. 264). Ses enluminures furent exécutées entre 1338 et 1344. Il fut utilisé par Bengt Thordeman, l'auteur du rapport de fouille, pour son étude sur les armures de Visby ${ }^{3}$. Ce manuscrit fut enluminé à une période charnière dans l'histoire de l'équipement et de l'armement médiéval, qui voit notamment le bassinet occuper une place prédominante, au dépend du heaume. Celui-ci est bien présent dans La Romance

\footnotetext{
${ }^{2}$ Voir Ada BrUHn HoffMEYER, Middelalderens Tveaeggede Svaerd, Københaven, Udgivet Af Tøjhusmuseet, 2 vol., 1954.

${ }^{3}$ Bengt ThORDEMAN, op. cit., p. 211 sqq.
} 
d'Alexandre mais le port du bassinet à la guerre prendra de plus en plus le pas sur celui du heaume.

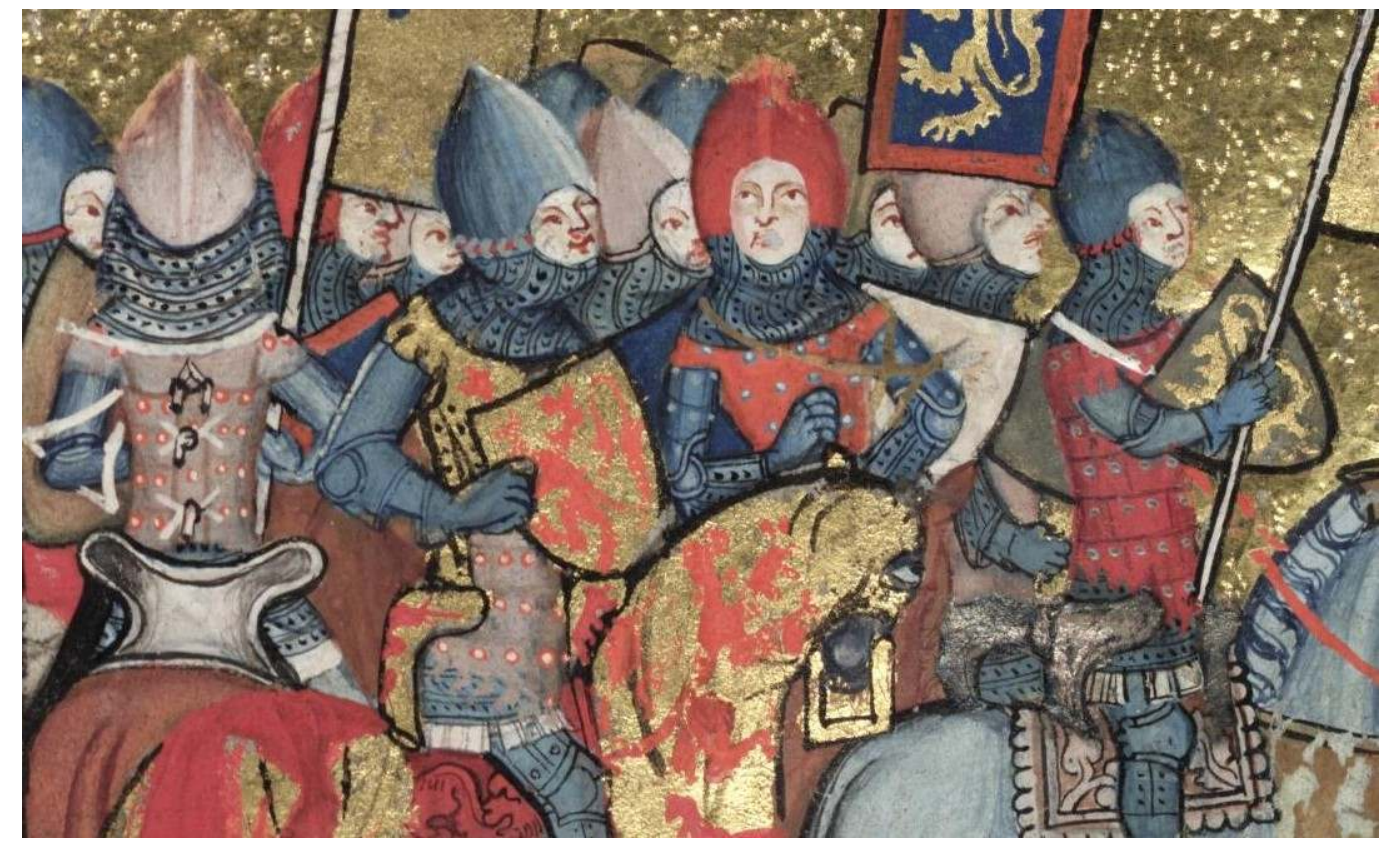

Illustration 4 : Hommes d'armes, détail, dans La Romance d'Alexandre, Oxford, Bodleian Library, ms. 264, $\mathrm{f}^{\circ} 66 \mathrm{r}^{\circ}$

Les personnages représentés sur les illustrations 4 et 5 sont dotés de cottes de plaques dont on distingue bien les alignements de rivets, fixant les plaques de métal sur le support. Une cotte d'arme pouvait être portée sur l'ensemble. Le camail de maille (coiffe de maille indépendante couvrant la tête et / ou tout ou partie des épaules. Pouvait être attachée au bassinet, comme on le voit sur l'image 4 et 5). On distingue également très bien, sur le personnage de gauche (image 5), les attaches dans le dos de l'armure ainsi que les guiges (sangles) maintenant l'écu (notamment celle passant autour du cou). Un haubergeon était également porté sous l'armure (on le distingue au niveau de la taille). 


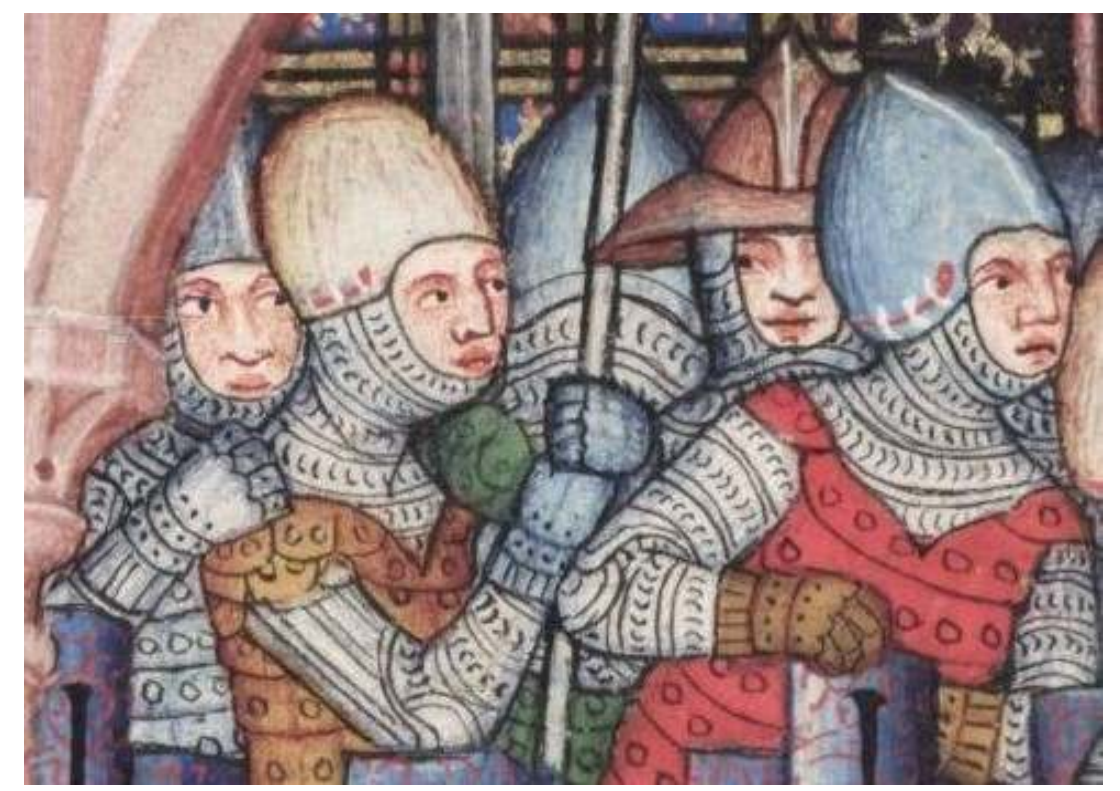

Illustration 5 : Hommes d'armes, détail, dans La Romance d'Alexandre, Oxford, Bodleian Library, ms. 264, $\mathrm{f}^{\circ} 58 \mathrm{r}^{\circ}$

On note l'absence de mézail (partie mobile du bassinet). Cependant, on peut en voir sur d'autres enluminures du même manuscrit, ainsi que d'autres types de casques («chapels de fer » et heaumes). Cela relève probablement d'un choix artistique de l'auteur car, comme le disait Claude Gaier : «nous devons renoncer à parler de la visière appliquée sur l'ouverture faciale du bassinet. Si son usage ne fait pas de doute, l'art figuratif évite de le représenter ou même d'en suggérer la présence par celle des charnières. ॥»

Les gantelets (illustration 5) sont très similaires à ceux découverts à Visby. Par la suite, les gants de plaques tendront vers un plus petit nombre de pièces, ils perdront en souplesse ce qu'ils gagneront en solidité.

$* * * *$

\footnotetext{
${ }^{4}$ Voir Claude GAIER, «L'évolution et l'usage de l'armement personnel défensif au pays de Liège du XII ${ }^{\mathrm{e}}$ au XIV ${ }^{\mathrm{e}}$ siècle ", dans Armes et combats dans l'univers médiéval, t. I, De Boeck Université (coll. Bibliothèque du Moyen Âge), 2004, ici p. 140.
} 
Le second manuscrit, qui contient L'Histoire Romaine (aujourd'hui sous la cote Paris, Bibliothèque Sainte-Geneviève, 0777), est à peine plus tardif (vers 1370). Ignoré par B. Thordeman ${ }^{5}$, le rapprochement entre les armures de Visby et ce manuscrit n'avait jusqu'alors jamais été fait.

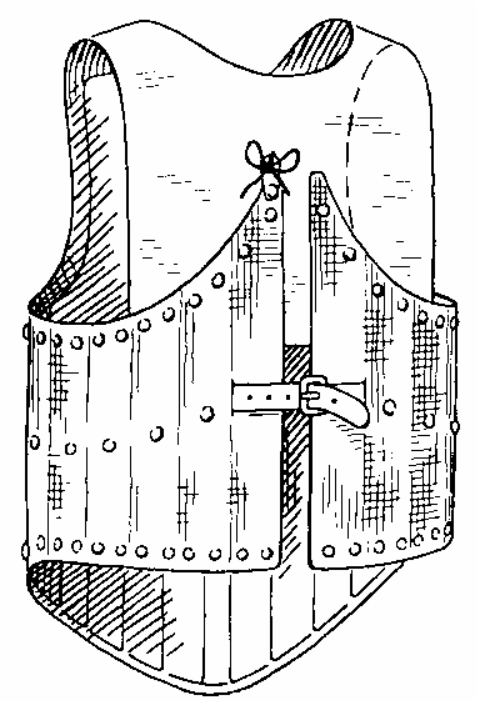

Illustration 6 : reconstitution d'une armure (Thordeman, 2001) ${ }^{6}$

L'illustration 6 présente une reconstitution d'un modèle mis au jour en fouille, qui est très proche de ceux dépeints sur les enluminures de L'Histoire Romaine (illustrations 7 et 8 ). Elles ont un intérêt tout particulier car elles nous montrent le dos des cottes de plaques dont sont équipés les combattants (piétons et cavaliers). Or, cette partie de l'armure est rarement représentée.

\footnotetext{
${ }^{5}$ Bengt ThORDEMAN, op. cit.

${ }^{6}$ Ibid., p. 222.
} 

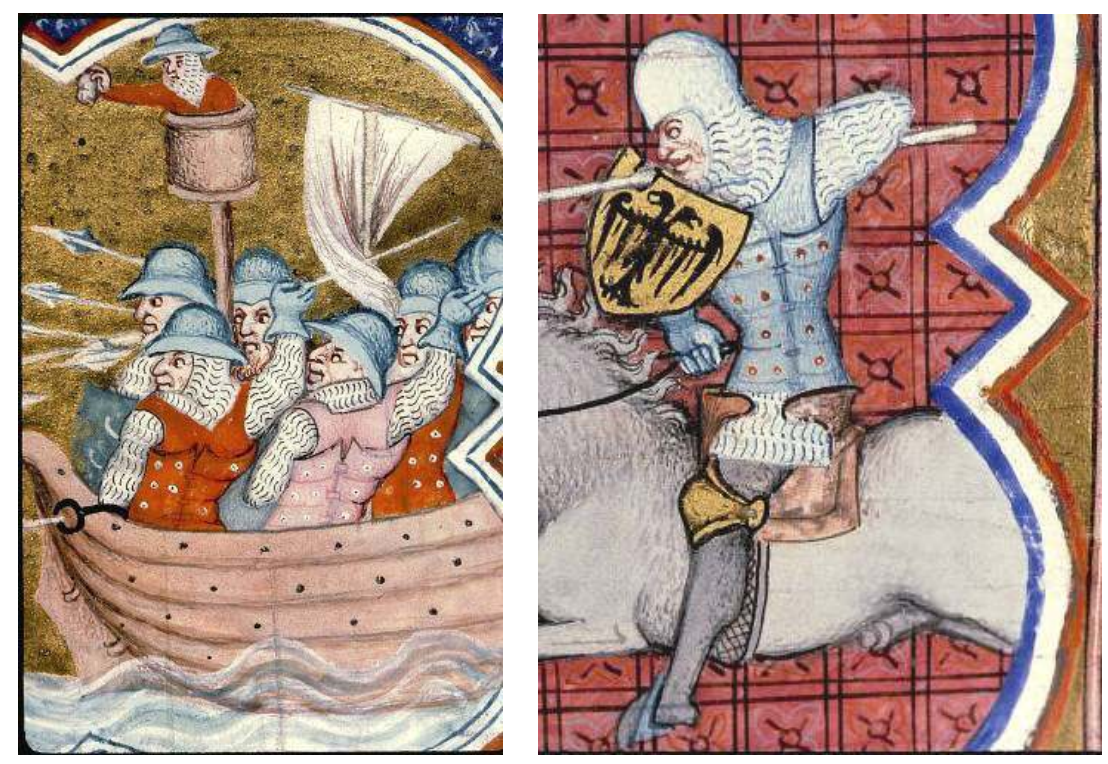

Illustrations 7 et 8 : Hommes d'armes, détails, dans L'Histoire Romaine, Paris, Bibliothèque Sainte-Geneviève, ms. 0777, $f^{\circ} 274 v^{\circ}$ et $f^{\circ} 119 v^{\circ}$

La configuration, les fixations et l'aspect général soutiennent ici la comparaison avec les découvertes archéologiques. Sous l'armure, on distingue le haubert, complément indispensable. Les soldats disposent également de gantelets et sont coiffés de « chapels de fer».

Nul doute qu'il existe d'autres manuscrits pouvant permettre le même type de rapprochement.

$* * * *$

\section{LES STIGMATES DE LA GUERRE}

Après avoir abordé le côté matériel, nous allons maintenant nous intéresser brièvement au côté humain, en examinant les blessures reçues par les combattants.

Mais avant de faire un survol des stigmates du combat médiéval, il faut garder à l'esprit que seules les blessures les plus profondes, celles laissant une empreinte sur l'os, nous sont parvenues. Il est difficile de savoir, par exemple, quelle pouvait être la proportion des blessures dont on a perdu la trace. 
Malgré tout, voilà ce qu'il est possible de dire. On distingue trois catégories de traumas: ceux produits par des armes tranchantes, perforantes ou contondantes.

La première, celle des armes tranchantes, est la plus fréquente à Visby avec 456 cas de blessures répertoriés. Elles vont de l'entaille légère à la section complète de l'os. En règle générale, elles sont assez simples à identifier car elles laissent des marques caractéristiques.

Les blessures par armes perforantes sont également faciles à identifier même si, quelquefois, il est difficile de dire par quel type d'arme elles ont été produites : un trou dans un crâne peut avoir été fait par la pointe d'une arme d'hast, d'une épée, ou d'un fer de trait.

Les blessures par armes contondantes sont quant à elles difficiles à identifier et seules quatre blessures de ce type purent être identifiées de manière évidente.

Une même arme pouvait infliger différents types de blessures : tranchante et perforante (voir même contondante) pour une épée, perforante et contondante pour un marteau d'arme, etc. À la guerre, plus l'arme était versatile, plus elle était efficace.

Lors d'un combat de mêlée, la première tâche était de rester en vie, la deuxième était de frapper dès que l'occasion se présentait. Toutes les parties du corps étaient susceptibles d'être touchées. La tête était souvent la cible prioritaire mais c'était aussi une des mieux protégées, on n'hésitait donc pas à frapper les mains, les bras, les jambes ou tout autre partie à sa portée. Ainsi, à Visby, $15 \%$ seulement des blessures répertoriées affectent les bras. L'usage de bouclier y est peut-être pour quelque chose.

Les jambes furent par contre une cible de choix, et particulièrement le tibia. On remarque aussi que le tibia gauche était plus souvent touché que le droit, ce qui est cohérent avec une situation de mêlée : lors d'une attaque, un 
combattant droitier avancera le plus souvent sa jambe gauche en premier, elle sera donc la plus exposée. Dans huit cas, le tibia fut sectionné en un coup.

Le fémur est le deuxième os de la jambe le plus touché. Quarante empreintes furent relevées sur des fémurs, dont treize cas où l'os est partiellement entamé et un cas où il est sectionné en une fois, ce qui laisse songeur quant à la violence du coup !

D'autres cas spectaculaires furent relevés concernant les membres inférieurs. Ainsi, on a retrouvé un individu dont la jambe droite fut tranchée net, juste au-dessus du pied et un autre qui avait pour sa part perdu les deux jambes, sectionnées à mi-tibia par un seul coup.

Concernant les traumas crâniens, près de la moitié des coups touchent l'os pariétal (illustration 9), et ce deux fois plus souvent à gauche qu'à droite, ce qui, là encore, est cohérent avec un affrontement de type face à face. En deuxième position viennent les coups à l'os frontal puis ceux touchant la partie occipitale du crâne, indiquant que la victime tournait alors le dos à son agresseur. Sur 241 blessures, 59 sont mixtes, alliant coupures et blessures par projectiles.

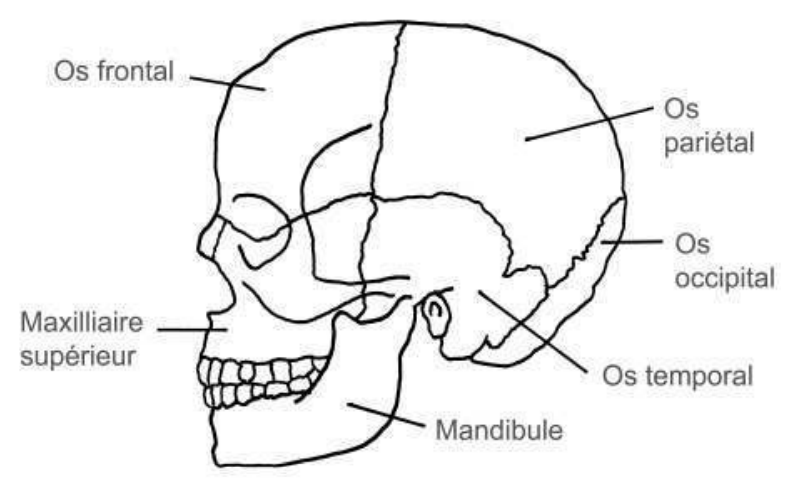

Illustration 9 : les os du crâne

D'une manière générale, les blessures à la face sont rares. Quatre cas de nez tranchés furent relevés ainsi que 15 blessures à la mâchoire inférieure (dont neuf ont tranché la mandibule). 
Parmi les traumas les plus impressionnants, on peut également citer plusieurs crânes dont le camail a été endommagé (" cut to pieces » pour reprendre l'expression employée par Thordeman ${ }^{7}$ ) et où le coup a pénétré l'os.

Il semble donc que de tels coups pouvaient endommager une cotte de maille, ce que certains chroniqueurs, comme Jean d'Outremeuse (contemporain de la bataille de Visby), avaient déjà signalés : « Il luy desmailhat sa brongne et le navrat en costeit...» ${ }^{8}$

D’une manière générale, le côté gauche est plus souvent touché que le droit. En fait, même si, dans un face à face entre deux droitiers, les coups portés à la gauche de l'adversaire viendront plus naturellement, ils ne sont en aucun cas limités à ce côté. Les revers étaient également très efficaces, surtout avec une épée à double tranchant.

Dans les trois quarts des cas, les coups sont portés obliquement du dessus (notamment ceux concernant le crâne). Viennent ensuite les coups verticaux. Les coups horizontaux et les coups ascendants sont rares. Les premiers sont difficiles à placer en mêlée si des compagnons sont à vos côtés, le mouvement demandant beaucoup de place pour être exécuté. Quant aux coups ascendants, ils sont plus techniques et difficiles à placer, surtout si l'on considère que le sol d'un champ de bataille s'encombre de corps et de divers objets dans les premières minutes du combat et qui sont autant d'obstacles potentiels pour un combattant effectuant un tel mouvement.

Les coups d'estoc devaient également être nombreux car ils présentent le meilleur compromis entre efficacité et mise en danger : c'est en effet un des premiers principes de toute escrime, qui, pour reprendre le mot de Molière, est «l'art de donner sans jamais recevoir. »

Rapides à effectuer, difficiles à parer, ils sont également moins susceptibles de laisser des traces sur un os. Enfin, un combattant intégré dans

\footnotetext{
${ }^{7}$ Berg ThORDEMAN, op. cit., p. 165.

${ }^{8}$ Voir Claude GAIER, « Armes et combats », art. cit., p. 361.
} 
une ligne ne peut faire que deux mouvements de manière efficace : une attaque verticale de haut en bas ou un estoc. À ce propos, Gilles de Rome écrivait à la fin du XIII ${ }^{\mathrm{e}}$ siècle « qu'il vaut mieux en bataille ferir d'estoc que de trenche. »"

On a vu que les projectiles avaient joués un grand rôle dans cette bataille, au vu du nombre de blessures occasionnées par les traits. Quelques cas de crânes ayant chacun cinq, six ou même sept blessures par projectiles furent retrouvés.

On estime que près de dix pour cent des combattants furent mis hors de combat par un trait à la tête. Une forte proportion donc, sachant que la tête est souvent bien protégée face à un tir (camail, casque, bouclier) et qu'elle représente une cible difficile à atteindre. Les effets de tels tirs sur des troupes inexpérimentées ont sans doute joué un rôle décisif dans le déroulement de la bataille. On sait que le moral tient une part extrêmement importante lors de ce type d'affrontement et que celui-ci est d'autant plus élevé que les troupes sont aguerries.

Végèce écrivait au sujet de la bataille rangée qu'en elle "triomphent l'habileté que donne l'expérience, la connaissance de la guerre $»^{10}$.

Que quelques sections des batailles danoises aient été désorganisées ou prises de paniques suite à ces tirs et c'en était fini car, comme devait le savoir les soldats danois, la panique est souvent la première cause de la défaite : elle se transmet rapidement et il est, sur le moment, impossible de récupérer ses troupes, surtout si elles sont inexpérimentées. C'est alors la curée, la cavalerie peut rattraper les fuyards pendant que les hommes à pieds réduisent les derniers foyers de résistance.

\footnotetext{
${ }^{9}$ Cité dans Christiane RaynaUd, Images et pouvoirs au Moyen Âge aux XIII ${ }^{e}-X V^{e}$ siècles, Paris, Le Léopard d'Or, 1993, p. 180.

${ }^{10}$ Cité dans VÉGÈCE, Traité de l'art militaire, Paris, J. Corréard, traduction de Victor DEVELAY, 1859, p. 152.
} 
On le voit, l'étude des champs de batailles et de leurs charniers pourrait apporter énormément à l'étude de l'art de la guerre au Moyen Âge.

De même, une recherche et une exploitation systématique des fonds iconographiques ferait sans aucun doute ressurgir des éléments concernant non seulement la guerre mais également la phase qui la précède : son apprentissage par le biais du duel (escrime civile), des joutes et de diverses pratiques dédiées que laisse déjà apparaître certains manuscrits. À cet égard, le manuscrit 264 de la Bodleian Library a encore beaucoup de choses à dire mais cela fera peut être l'objet d'une publication ultérieure.

Il est très dommage en tout cas que cette discipline, très développée chez nos confrères Anglo-Saxons, et connue sous le nom de «battlefield archaeology », ne connaisse pas le même engouement en France.

\section{Autres références :}

Philippe Contamine, La guerre au Moyen Âge, Paris, Presses Universitaires de France (6º́dition, janvier 2003), 1980.

Éric CRUBEZY et Jean-Claude HÉLAS, «Le combattant à l'époque médiévale : vers une approche archéologique et paléopathologique », dans Le combattant au Moyen $\hat{A} g e$. Actes du Congrès de la Société des historiens médiévistes de l'enseignement supérieur public (Montpellier, 1987), Paris, Publications de la Sorbonne, 1995, p. 297 $s q q$.

Alain Mounier KuHN, « Les blessures de guerre et l'armement au Moyen Âge dans l'Occident latin », Médiévales, 39 (janvier 2001), p. 112-136.

Pamela PORTER, «The Ways of War in Medieval Manuscript Illumination: Tracing and Assessing the Evidence », dans Armies, Chivalry and Warfare in medieval Britain and France, Proceedings of the 1995 Harlaxton Symposium, Harlaxton Medieval Studies, VII, Paul Watkins, Stamford, 1998.

Pierre-André SIGAL, « Les coups et les blessures reçus par le combattant à cheval en occident aux $\mathrm{XII}^{\mathrm{e}}$ et $\mathrm{XIII}^{\mathrm{e}}$ siècles » dans Le combattant au Moyen Age. Actes du 
Congrès de la Société des historiens médiévistes de l'enseignement supérieur public (Montpellier, 1987), Paris, Publications de la Sorbonne, 1995, p. 171 sqq. 